\title{
Oxycodon/Naloxon bei chronischen Schmerzen effektiv und kostensparend
}

_ Die Schmerztherapie mit Targin ${ }^{\circledast}$ (Oxycodon/Naloxon) ist hinsichtlich Analgesie und Lebensqualität gut wirksam, verursacht aber geringere Kosten als eine Behandlung mit anderen starken Opioiden. Das zeigt eine nicht-interventionelle Versorgungsstudie zur Therapie chronischer Rückenschmerzen des Instituts für Empirische Gesundheitsökonomie, deren Zwischenauswertung Studienleiter Reinhard Rychlik, Burscheid, präsentierte. 924 Patienten wurden über zwölf Monate mit der Fixkombination aus retardiertem Oxycodon und retardiertem Naloxon oder mit anderen starken Opioiden behandelt. Die
Kombination steigerte die gesundheitsbezogene Lebensqualität signifikant besser als die alternativen Opioide: Gegenüber den Ausgangswerten verbesserten sich körperliche Schmerzen um mehr als 100 vs. $43,2 \%$ ), körperliche Funktion um 74,7\% (vs. 28,3\%), Vitalität um $51,4 \%$ (vs. 17,5) und soziale Funktion um $41,8 \%$ (vs. 13,4\%).

Die Schmerzintensität beurteilten die Patienten im BPI-SF (Brief Pain Inventory - Short Form) auf einer Skala von 0 bis 40 (stärkster Schmerz): Oxycodon/Naloxon reduzierte die Intensität um $45 \%$ (vs. 24,7\%) - dabei waren die Ausgangswerte in dieser Gruppe höher, die Werte am Studienende deutlich niedriger als in der Kontrollgruppe.

Die direkten Kosten der Targin ${ }^{\circledast}$-Therapie waren um etwa 370 Euro geringer (2.403 vs. 2.773 Euro). Sie reduzierten sich vor allem infolge selteneren Gebrauchs von Ko- und Rescue-Medikation, Notfallbehandlung und Klinikaufenthalt. Eine Kosten-Effektivitätsanalyse fiel damit zugunsten Oxycodon/ Naloxon aus: „Bessere Wirksamkeit zu geringeren Kosten", fasste Rychlik zusammen.

Expertengespräch „Schmerztherapie im Spannungsfeld zwischen Lebensqualität und Kostendruck - Neue Studiendaten zu Targin ${ }^{\circledR}$, im Rahmen des 22. Deutschen interdisziplinären Schmerz- und Palliativkongresses, Frankfurt, 23. März 2011, Veranstalter: Mundipharma; Bericht: Michael Koczorek, Bremen

\section{Chronifizierung postoperativer Schmerzen verhindern}

gewiesen hat. Neben der guten schmerzlindernden Wirkung ist der anxiolytische Effekt ein Vorteil der Substanz.

- Chronische Schmerzsyndrome kommen gehäuft nach Mamma-Operation, Cholezystektomie oder Thorax-Herzchirurgie vor. Bei über der Hälfte aller Patienten mit hernienchirurgischem Eingriff persistieren die Schmerzen nach einem Jahr; bis zu 80\% aller Patienten mit Amputation leiden unter Phantomschmerz. "Drastische Zahlen", kommentierte Thomas Cegla, Wuppertal, angesichts von hierzulande 45 Millionen medizinischen Eingriffen, von denen etwa ein Drittel Operationen sind. Vor allem bei insuffizienter schmerztherapeutischer Behandlung drohten die Schmerzen zu chronifizieren, so Cegla. Zusätzlich zu Trauma und Gewebsverletzung durch Operation oder Unfall verstärken eine Reihe weiterer Faktoren das Risiko: Die psychische Belastung in der Krankenhaussituation oder psychische Begleiterkrankungen, etwa depressive Verstimmung, tragen dazu bei, körpereigene Regulationsmechanismen wie die absteigende Schmerzhemmung zu schwächen. Typische Symptome der neuropathischen Schmerzen sind Par- und Dysästhesien, Hyperpathie, Brennschmerz und einschießende Schmerzattacken, Dauerschmerzen und evozierte Schmerzen. Diese müssen nach eingehender Schmerzdiagnostik im Rahmen einer suffizienten multimodalen perioperativen Schmerztherapie behandelt werden. Für die Therapie neuropathischer Schmerzen stehen langwirksame Opioide, trizyklische Antidepressiva und
Antikonvulsiva zur Verfügung, die allein oder kombiniert eingesetzt werden können. Unter den Antikonvulsiva ist Pregabalin eine bewährte Option, die in verschiedenen placebokontrollierten Studien ihre Wirksamkeit nach-
Symposium „Chronischer Schmerz nach Nervenschaden - Intervention, Pharmakologie, Psyche", anlässlich des Deutschen Schmerzund Palliativtages, Frankfurt, 26. März 2011, Veranstalter: Pfizer; Bericht: Michael Koczorek, Bremen

\section{Vorsprung durch Fortbildung}

- Die Gesundheitslandschaft wandelt sich stetig. Know-how und Qualifikation sind entscheidend für den Erfolg jeder Arztpraxis. schwa-medico bietet mit seinem Fortbildungsprogramm Ärzten und ihren Mitarbeitern ein persönliches „Fitnessprogramm“. Das Angebot umfasst u.a. Seminare zur Schmerztherapie und zu IGeL in der Arztpraxis. Sie richten sich an Orthopäden, Sportmediziner, Physio- \& Schmerztherapeuten. Darunter:

Vibrationstherapie in der Praxis

(Fortbildungspunkte sind angefragt, Gebühr: 119,00€)

Referent: Dr. med. Rainer Klügel, Geilenkirchen

\section{Wann und wo:}

17. September 2011 in Potsdam oder am 26. November 2011 in Frankfurt
Neuroorthopädische Diagnostik und Therapie

(Gebühr: 119,00€)

_ Manuelle Diagnostik und Therapie von Rückenschmerzen - Muskelverspannungen erkennen und behandeln

- TENS und Vibrationstherapie, Theorie und Praxis

Referenten: Stefan Holthusen, Göttingen, und Dr. med. Martin Gessler, München Wann und wo:

9. Juli 2011 in Bad Herrenalb

Anmeldungen und weitere Informationen: schwa-medico - Medizinische Apparate Vertriebsgesellschaft $\mathrm{mbH}$ Borngasse 9, 35630 Ehringshausen Tel. 06443 / 8388-615; Fax 06443 / 8388-629 Ansprechpartnerin: Yvonne Greilich E-Mail:y.greilich@schwa-medico.de 